
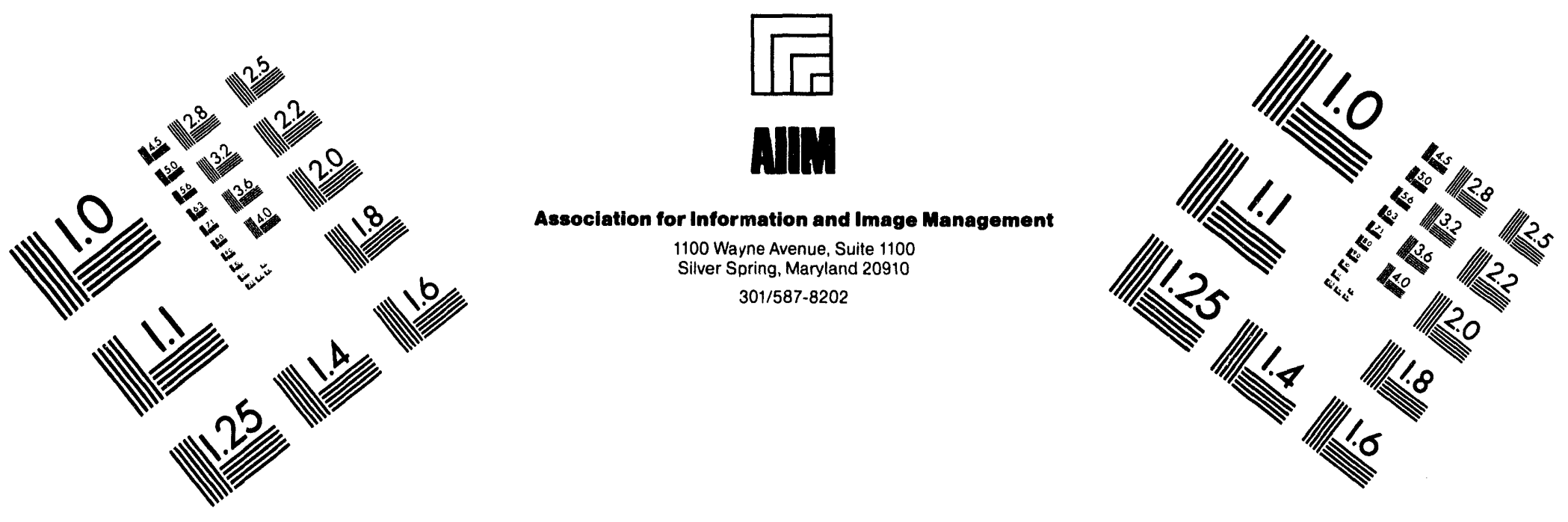

\title{
Centimeter
}

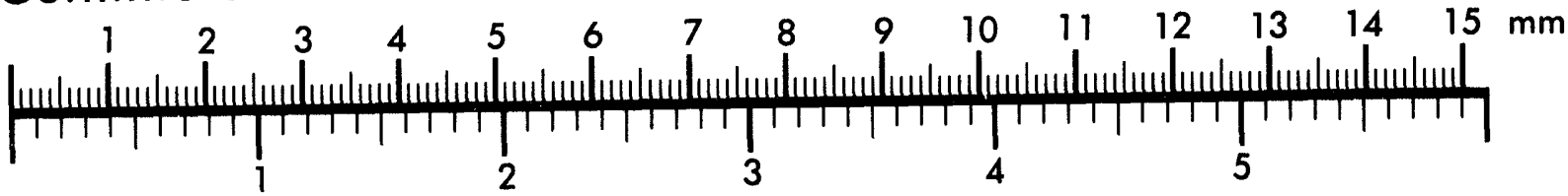
Inches
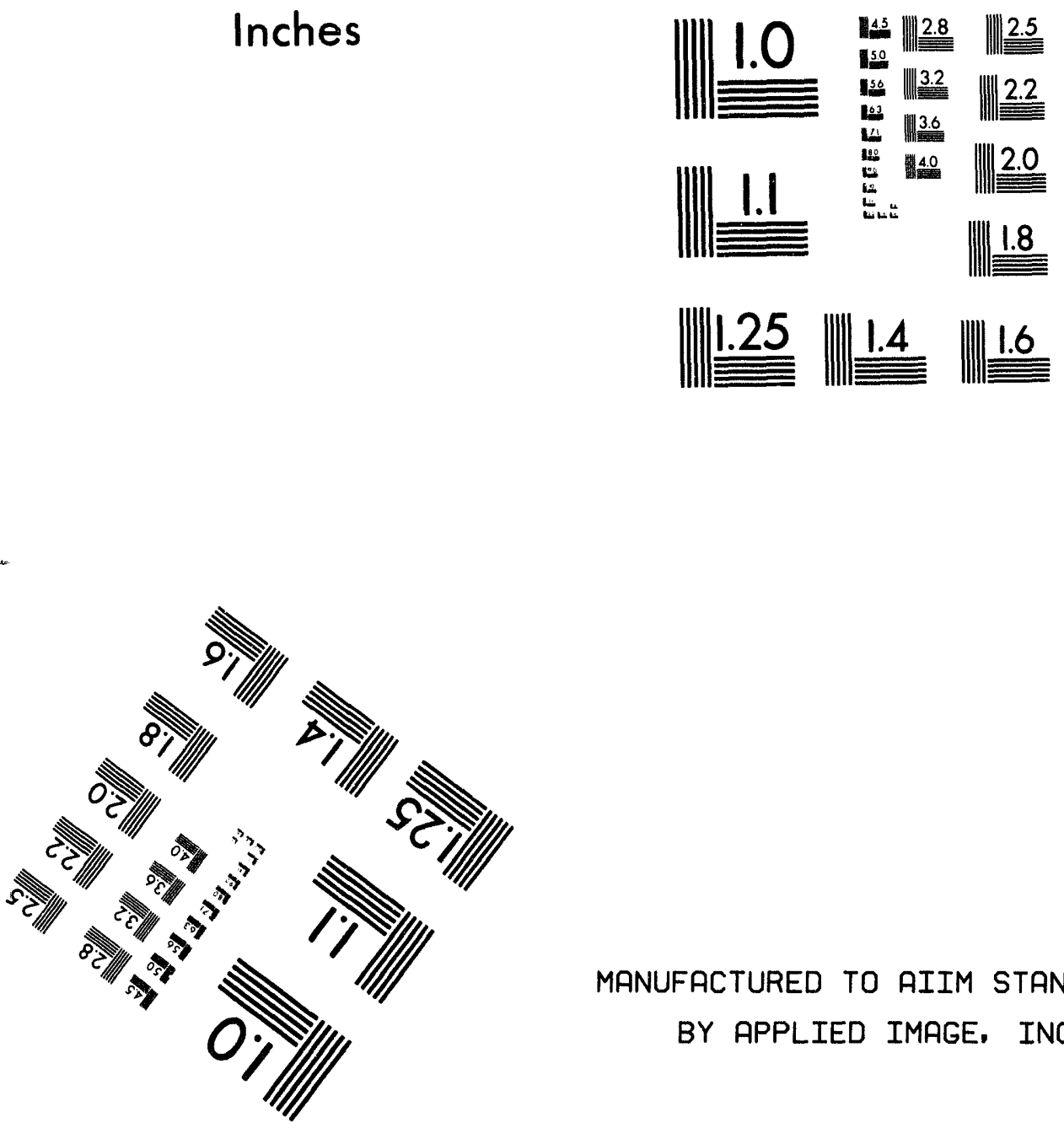

MANUFACTURED TO AIIM STANDARDS

BY APPLIED IMAGE, INC.

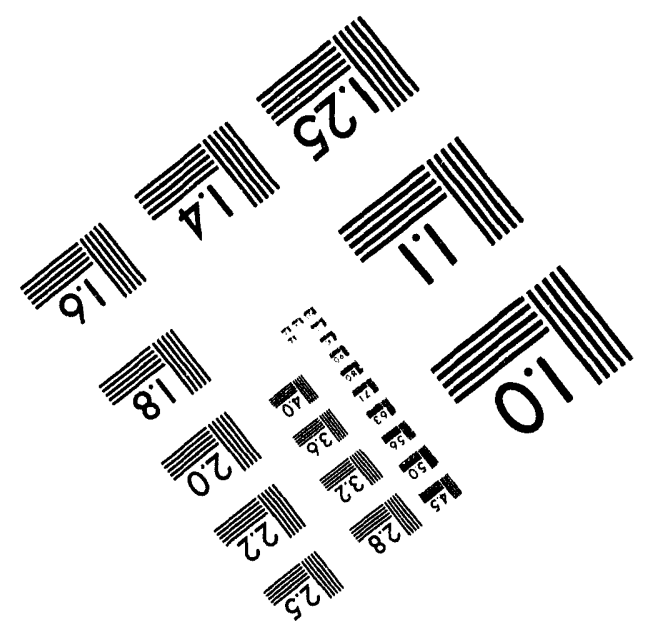



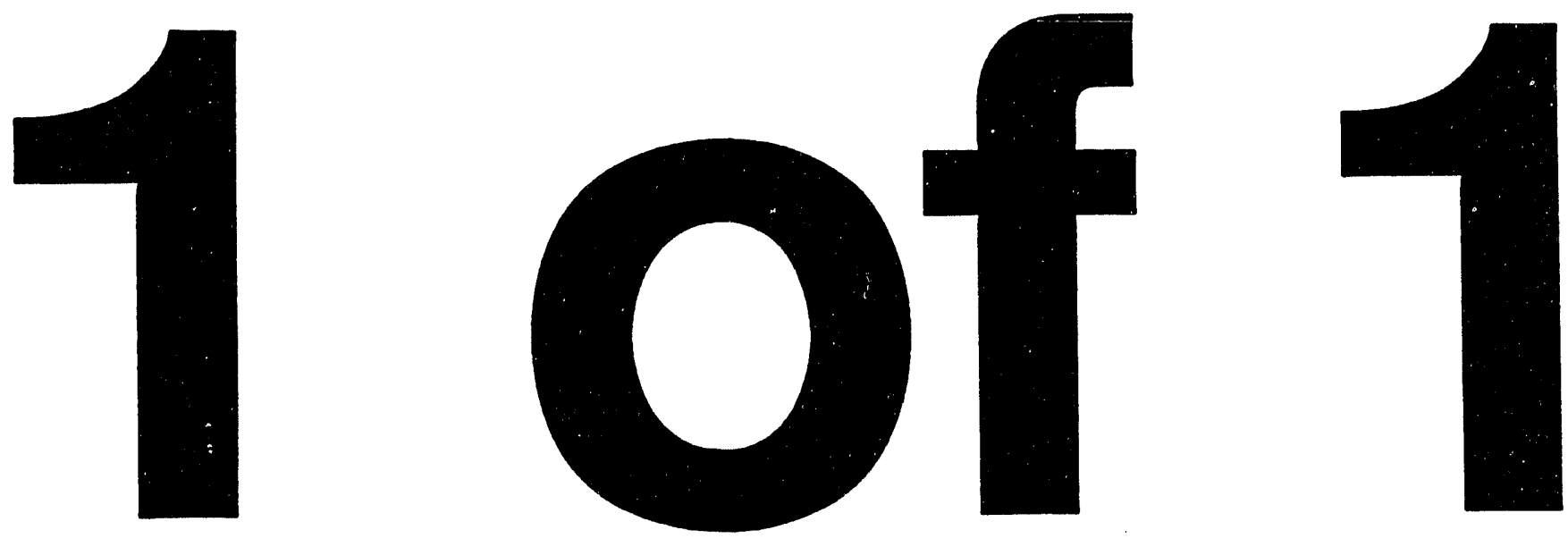
ORNL/TM-12764

Engineering Physics and Mathematics Division

\section{A MULTIPLE DIVIDE-AND-CONQUER (MDC) ALGORITHM FOR OPTIMAL ALIGNMENTS IN LINEAR SPACE}

X. Guan and E. C. Uberbacher

DATE PUBLISHED - June 1994

Research supported by the Office of Health and Environmental Research, U.S. Department of Energy, and the Laboratory Directed Research and Development Programs

\section{Prepared by the}

OAK RIDGE NATIONAL LABORATORY

Oak Ridge, Tennessee 37831

Managed by

MARTIN MARIETTA ENERGY SYSTEMS, INC. for the

U.S. DEPARTMENT OF ENERGY under Contract No. DE-AC05-84OR21400 


\section{CONTENTS}

Page No.

ABSTRACT v v

$\begin{array}{ll}\text { 1. Introduction } & 1\end{array}$

2. Dynamic Programming Algorithms 1

3. A New Linear Space Alignment Algorithm 3

$\begin{array}{ll}\text { 4. Analysis and Results } & 4\end{array}$

$\begin{array}{lr}\text { 5. Conclusions } & 6\end{array}$

$\begin{array}{ll}\text { Acknowledgments } & 7\end{array}$

$\begin{array}{lc}\text { References } & \mathbf{8}\end{array}$ 


\begin{abstract}
This paper describes an algorithm for optimal sequence alignments in linear space. A new multiple divide-and-conquer technique is presented that leads to a linear space alignment algorithm which improves upon an existing algorithm by Myers and Miller.
\end{abstract}




\section{Introduction}

Dynamic programming algorithms are often used to find the similarities of sequences as well as to deliver the actual alignment of two sequences. Two kinds of alignments are used to compare sequences: local alignments and global alignments. The local alignments attempt to locate conserved regions, while the global alignments identify overall relationship between two sequences.

While dynamic programming algorithms are relatively time consuming, the space required is often the limiting factor when aligning long sequences. A linear space algorithm for computing maximal common subsequences, proposed by Hirschberg [1], was applied by Myers and Miller [2] to deliver optimal alignments in linear space. We have improved the Myers and Miller algorithm by introducing a multiple divide and conquer technique that reduces the algorithm's running time while maintaining its linear space property.

In the following sections, we use brackets [] to represent a matrix and parentheses () to represent a single entry in the matrix: $D[m, n]$ represents a matrix $D$, while $D(i, j)$ represents a single entry in the matrix $D$ at the $i$ th row and the $j$ th column.

\section{Dynamic Programming Algorithms}

We first give a dynamic programming algorithm for global sequence alignments. Given two sequences $A=a_{1} a_{2} \ldots a_{m}$ and $B=b_{1} b_{2} \ldots b_{n}$, a cost, $w$, for $k$ insertions or deletions is defined as $w(k)=(u * k+v)$, where $u \geq 0$ and $v \geq 0$. The minimum cost of aligning the two sequences is given in [3]:

$$
D(i, j)=\min \begin{cases}D(i-1, j-1)+c\left(b_{i}, a_{j}\right), & \\ P(i, j), & 0<i \leq m, 0<j \leq n \\ Q(i, j) . & 0<1\end{cases}
$$

where

$$
\begin{gathered}
P(i, j)=\min \left\{\begin{array}{l}
D(i-1, j)+w(1), \\
P(i-1, j)+u
\end{array}\right. \\
Q(i, j)=\min \left\{\begin{array}{l}
D(i, j-1)+w(1) \\
Q(i, j-1)+u
\end{array}\right. \\
P(0, k)=Q(k, 0)=D(k, 0)=D(0, k)=w(k), \forall k>0
\end{gathered}
$$

and 


$$
c\left(b_{i}, a_{j}\right)= \begin{cases}\geq 0 & \text { if } b_{i} \neq a_{j} \\ \leq 0 & \text { if } b_{i}=a_{j}\end{cases}
$$

The value $D(m, n)$ represents the minimum cost of aligning the two sequences.

To deliver the optimal alignment (that corresponds to the minimal cost), a traceback procedure is performed to find the actual alignment. A straightforward implementation of the traceback procedure requires $O(n m)$ space, thus limiting it to only short sequence alignments.

If only the minimum cost is needed and not the alignment, linear space is enough to do the calculation. As the value in position $(i, j)$ depends only on values in three other positions, i.e., $(i, j-1),(i-1, j-1)$ and $(i-1, j)$, one can do the calculation in a row by row fashion using only linear space (see [2] for details on the linear space cost only algorithm). This algorithm will be referred to later as the linear-space-cost-only algorithm.

The Hirschberg algorithm, applied by Myers and Miller to sequence alignments [2], is a divide-and-conquer algorithm designed to compute the alignment using linear space. The idea is to divide the first sequence into two halves $A_{1} A_{2}$ and then to locate the optimal point in the second sequence (which divides the second sequence into two parts $B_{1} B_{2}$ ) such that the optimal alignment is the concatenation of the optimal alignment of $A_{1}$ and $B_{1}$ and the optimal alignment of $A_{2}$ and $B_{2}$ (see Figure 1).

\section{B1}

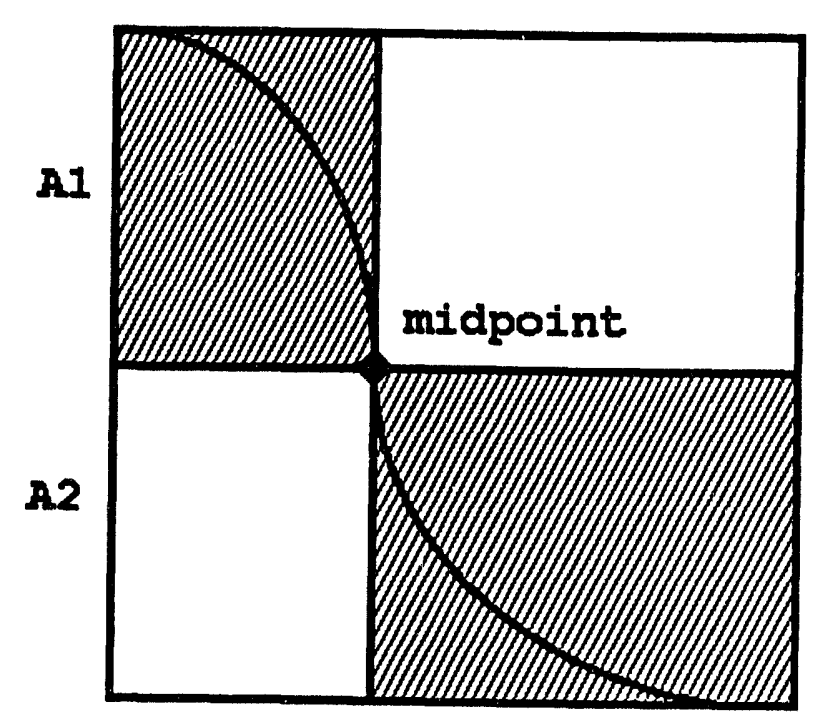

Figure 1. Divide and conquer. 
The optimal alignment of two sequences can be thought of as a path in the cost matrix that starts at $D(0,0)$ and ends at $D(m, n)$. This path must cross the middle row (midrow) $i^{*}=\frac{m}{2}$. The problem is to find the point (midpoint) where the optimal path crosses the midrow.

Myers and Miller's algorithm uses the linear-space-cost-only algorithm to find the midpoint. First it calculates

- $D F(j)=$ the minimum cost of aligning $a_{1} a_{2} \ldots a_{i *}$ and $b_{1} b_{2} \ldots b_{j}$

- $D R(j)=$ the minimum cost of aligning $a_{i *+1} \ldots a_{n-1} a_{m}$ and $b_{j+1} b_{j+2} \ldots b_{n}$

where $0 \leq j \leq n$. Then it calculates

$$
j^{*}=\min _{j \in[0, n]}\{D F(j)+D R(n-j)\} .
$$

$\left(i^{*}, j^{*}\right)$ is the optimal midpoint (for a proof see [1]).

The optimal alignment is obtained by recursively finding the optimal alignment of the two subsequences before the midpoint and the optimal alignment of the two subsequences after the midpoint.

The actual algorithm is a bit more involved as it considers the types of the operations (replacement, delete, etc.) before and after the midpoint on the optimal path, and divides the problem accordingly.

\section{A New Linear Space Alignment Algorithm}

We first introduce a new technique to find the midpoint. We define a matrix $C R O S S[m, n]$ : $\operatorname{CROSS}(i, j)$, when $i \geq \frac{m}{2}$, contains the column of the cost matrix where the optimal path that starts at $D(0,0)$ and ends at $D(i, j)$ crosses the midrow. CROSS $(i, j)$ is undefined for $i<\frac{m}{2}$. When both $D[m, n]$ and CROSS $[m, n]$ have been calculated, $D(m, j)$ contains the minimum cost of aligning $A=a_{1} a_{2} \ldots a_{m}$ and $B=b_{1} b_{2} \ldots b_{j}, 0 \leq j \leq n$, and $\operatorname{CROSS}(m, j)$ contains the column where the optimal path that starts at $(0,0)$ and ends at $(m, j)$ crosses the midrow. In particular, CROSS $(m, n)$ contains the midpoint.

The calculation of CROSS $[m, n]$ is straightforward. Suppose we are calculating $D(i, j)$. The last operation in Figure 2 refers to the operation (i.e., a replacement, a deletion, or a insertion) that leads to $D(i, j)$. The path in Figure 2 refers to the optimal path that starts at $D(0,0)$ and ends at $D(i, j)$. 
- If $\left(i=i^{*}+1\right)$ then

- CROSS $(i, j)=j$ if the last operation is a deletion,

$-\operatorname{CROSS}(i, j)=j-1$ if the last operation is a replacement,

- $\operatorname{CROSS}(i, j)=\operatorname{CROSS}(i, j-1)$ if the last operation is an insertion.

- else pass on the crossing-point information

- $\operatorname{CROSS}(i, j)=\operatorname{CROSS}(i-1, j)$ if the last operation is a deletion,

- $\operatorname{CROSS}(i, j)=\operatorname{CROSS}(i-1, j-1)$ if the last operation is a replacement,

- CROSS $(i, j)=\operatorname{CROSS}(i, j-1)$ if the last operation is an insertion.

Figure 2. Calculation of $\operatorname{CROSS}(i, j)$ at $D(i, j)$.

Like $D[i, j], C R O S S[m, n]$ can be calculated easily in linear space. All we need is the value CROSS $(m, n)$. The cost is the added operation and the extra $n$ space. We call the linear space cost only alignment algorithm with the added matrix $\operatorname{CROSS}[m, n]$ the modified-linearspace-cost-only alignment algorithm.

A natural extension of the above divide-and-conquer technique is to use more than one dividing row. The calculations of where the optimal path crosses the dividing rows are similar to that for midrow. Extra $k n$ spare is need to store the crossing information for $k$ dividing rows .

The main MDC algorithm is outlined as follows: given two sequences,

- apply the modified-linear-space-cost-only alignment algorithm to find and save the crossing information at these $k$ dividing rows,

- recursively divide each sequence into $k$ subsequences according to the crossing information at these $k$ dividing rows until the alignment is trivial to do,

- the optimal alignment is the concatenation of the optimal alignments of these $k$ pairs of subsequences.

\section{Analysis and Results}

Let $T_{1}$ be the time of linear-space-cost-only algorithm. The total area of the calculation of the linear-space-cost-only algorithm is the cost matrix $D$. Using the Myers and Miller algorithm, after one recursion, the problem is divided into two subproblems whose total area is half of the cost matrix. In turn these two subproblems are divided into four subproblems whose total area is one fourth of the cost matrix, and so on (see Figure 3(a)). 


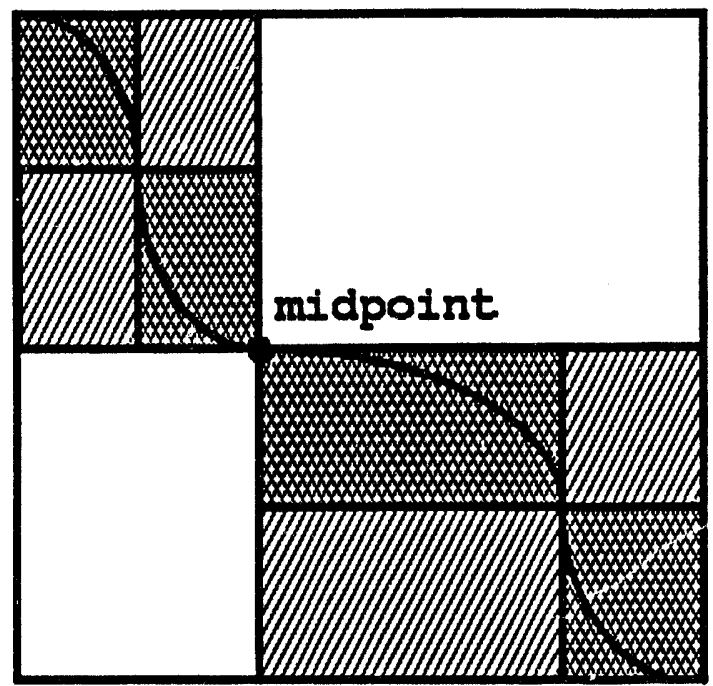

(a) The Myers and Miller algorithm.

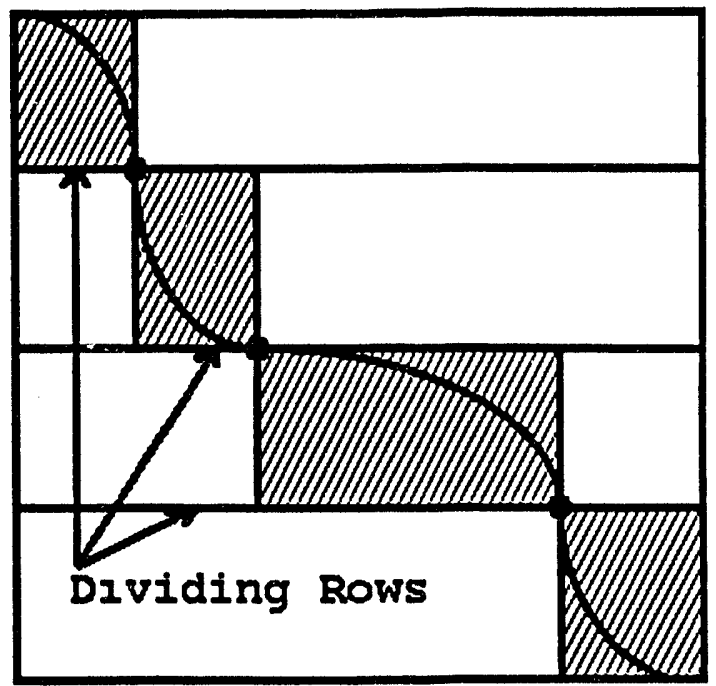

(b) The MDC algorithm.

Figure 3. Divide and conquer.

So the approximate time $T_{2}$ for the Myers and Miller algorithm is

$$
T_{2}=T_{1} *\left(1+\frac{1}{2}+\frac{1}{4}+\cdots\right) \approx 2 T_{1}
$$

If four dividing rows are used in the $\mathrm{MDC}$ algorithm, each recursion divides a problem (or a subproblem) into four subproblems (see Figure 3(b)).

So the time of our algorithm $T_{3}$ is approximately

$$
T_{3}=T_{1} *\left(1+\frac{1}{4}+\frac{1}{4^{2}}+\cdots\right) \approx \frac{4}{3} T_{1}
$$

In general, let $d$ be the number of dividing rows. A problem is recursively divided into $s=d+1$ subproblems, so $T_{3}$ is

$$
T_{3} \approx \frac{s}{s-1} T_{1}
$$

This is only an estimate since the cost of the added operation is not taken into account. But as demonstrated below, the gain as a result of the introduction of the multiple divideand-conquer technique is much more than the cost, resulting in substantial saving in the execution time. 
To test the method, we used a simplified version, where $w(k)=u * k$. In the following table, MDC4, MDC8, and MDC16 refer to the MDC algorithm when $s=4,8$, or 16 respectively, and seq len is the length of each of the two sequences. The algorithms were tested on a SUN SPARC 10 Workstation. We used a straightforward implementation of the algorithm. A refined version that reduces the cost of the newly introduced operation in the linear-spacecost-only algorithm will lead to a faster algorithm.

\begin{tabular}{|r|r|r|r|r|}
\hline seq_en & Myers & MDC4 & MDC8 & MDC16 \\
\hline 3500 & $0: 34$ & $0: 29$ & $0: 25$ & $0: 23$ \\
\hline 7000 & $2: 41$ & $1: 55$ & $1: 41$ & $1: 35$ \\
\hline 14000 & $9: 13$ & $7: 45$ & $7: 07$ & $6: 21$ \\
\hline 28000 & $37: 05$ & $31: 40$ & $27: 05$ & $26: 08$ \\
\hline
\end{tabular}

Table 2. Times (in minutes:seconds) of the linear space alignment algorithms

\section{Conclusions}

Sequence alignments using dynamic programming algorithms are demanding in both time and space, so efficient sequence alignment algorithms have been an active topic in computational biology. Here we have presented a multiple divide-and-conquer technique which improves the linear space alignment algorithm proposed by Myers and Miller. Still, aligning long sequences takes considerable time, so approximate algorithms which use prescreening methods such as dot matrix and $k$-tuple look tables, may be used together with this optimal dynamic programming algorithm to produce even more efficient systems. 


\section{Acknowledgments}

This Research was supported by the Office of Health and Environmental Research, U.S. Department of Energy under Contract No. DE-AC05-84OR21400 with Martin Marietta Energy System, Inc. 


\section{References}

[1] D. S. Hirschberg, "A Linear Space Algorithm for Computing Maximal Common Subsequences," Communications of the ACM, vol. 18, 1975, 341-343.

[2] E. W. Myers and W. Miller, "Optimal Alignments in Linear Space," CABIOS, vol. 4, 1988, 11-17.

[3] O. Gotoh, "An Improved algorithm for matching biological sequences," Journal of Molecular Biology, vol. 162, 1982, 705-708. 


\section{INTERNAL DISTRIBUTION}

1. B. R. Appleton

2. M. Beckerman

3. J. R. Einstein

4. C. W. Glover

5. W. C. Grimmell

6-10. X. Guan

11. J. P. Jones

12. H. E. Knee

13-17. R. C. Mann

18. S. Matis

19-20. R. J. Mural

21. E. M. Oblow

22. C. E. Oliver
23. S. Petrov

24. N. S. V. Rao

25. D. B. Reister

26. M. B. Shah

27-31. E. C. Uberbacher

32. R. C. Ward

33. $\mathrm{X}$. Ying

34. EPMD Reports Office

35-36. Laboratory Records Department

37. Laboratory Records, ORNL-RC

38. Document Reference Section

39. Central Research Library

40. ORNL Patent Office

\section{EXTERNAL DISTRIBUTION}

41. Office of Assistant Manager for Energy Research and Development, Oak Ridge Operations, U.S. Department of Energy, P.O. Box 2008, Oak Ridge, TN 37831

42. Jim Decker, Director, Office of Energy Research, Dept. of Energy, Washington, DC 20585

43. David Smith, Health Effects \& Life Sciences Research Division, Office of Health \& Environmental Research, Dept. of Energy, Washington, DC 20585

44. B. J. Barnhart, Health Effects and Life Sciences Research Division, Office of Health and Environmental Research, Dept. of Energy, Washington, DC 20585

43. John Wooley, Health Effects and Life Sciences Research Division, Office of Health and Environmental Research, Dept. of Energy, Washington, DC 20585

44. Mark S. Boguski, National Institutes of Health NCBI/NLM, 8600 Rockville Pike, Bethesda, MD 20894

45. M. D. Zorn, Lawrence Berkeley Laboratory, MS 50B-3216, 1 Cyclotron Road, Berkeley, CA 94720

46. J. Fickett, Los Alamos National Laboratory, P.O. Box 1663, Los Alamos, NM 87545

47. Christian Burks, Los Alamos National Laboratory, Theorectical Biol. \& Biophysics Grp., T-10, MS K710, Los Alamos, NM 87545

48. A Jamie Cuticchia, John Hopkins Hosp./Welch Med. Library, 1830 East Monument Street, Baltimore, MD 21205-2100

49. E. Branscomb, Human Genome Center, Biomedical Sciences Division, Lawrence Livermore National Laboratory, Livermore, CA 94550

50. A. Lapedes, Center for Human Genome Studies, Los Alamos National Laboratory, P.O. Box 1663, Los Alamos, NM 87545

51. T. Hunkapiller, Division of Biology, California Institute of Technology, Pasadena, CA 91125

52. Radoje Drmanac, Argonne National Laboratory, 9700 S. Cass Avenue, Argonne, IL 60439 
53. Chris Fields, The Institute For Genomic Research, 932 Clopper Road, Gaithersburg, MD 20878

54. Office of Scientific and Technical Information, P.O. Box 62, Oak Ridge, TN 37831

55. Raymond F. Gesteland, University of Utah, Howard Hughes Medical Institute, 6160 Eccles Genetics Building, Salt Lake City, UT 84112

56. Steven Henikoff, Fred Hutchison Cancer Research Ctr., HHMI-FHCRC, 1124 Columbia Street, Seattle, WA 98104

57. Tim Hunkapiller, California Institute of Technology, 139-74, Pasadena, CA 91125

58. Thomas G. Marr, Cold Spring Harbor Laboratory, P.O. Box 100, Cold Spring Harbor, NY 11724

59. Sylvia J. Spengler, Lawrence Berkeley Laboratory, Human Genome Center, MS 1-213, 1 Cyclotron Road, Berkeley, CA 94720

60. Marvin Stodolsky, U.S. Department of Energy, Office of Health \& Environmental Res., ER-72 GTN, Washington, DC 20545-0001

61. Jude Shavlik, Computer Science, University of Wisconsin, 1210 W. Dayton Street, Madison, WI 53706 

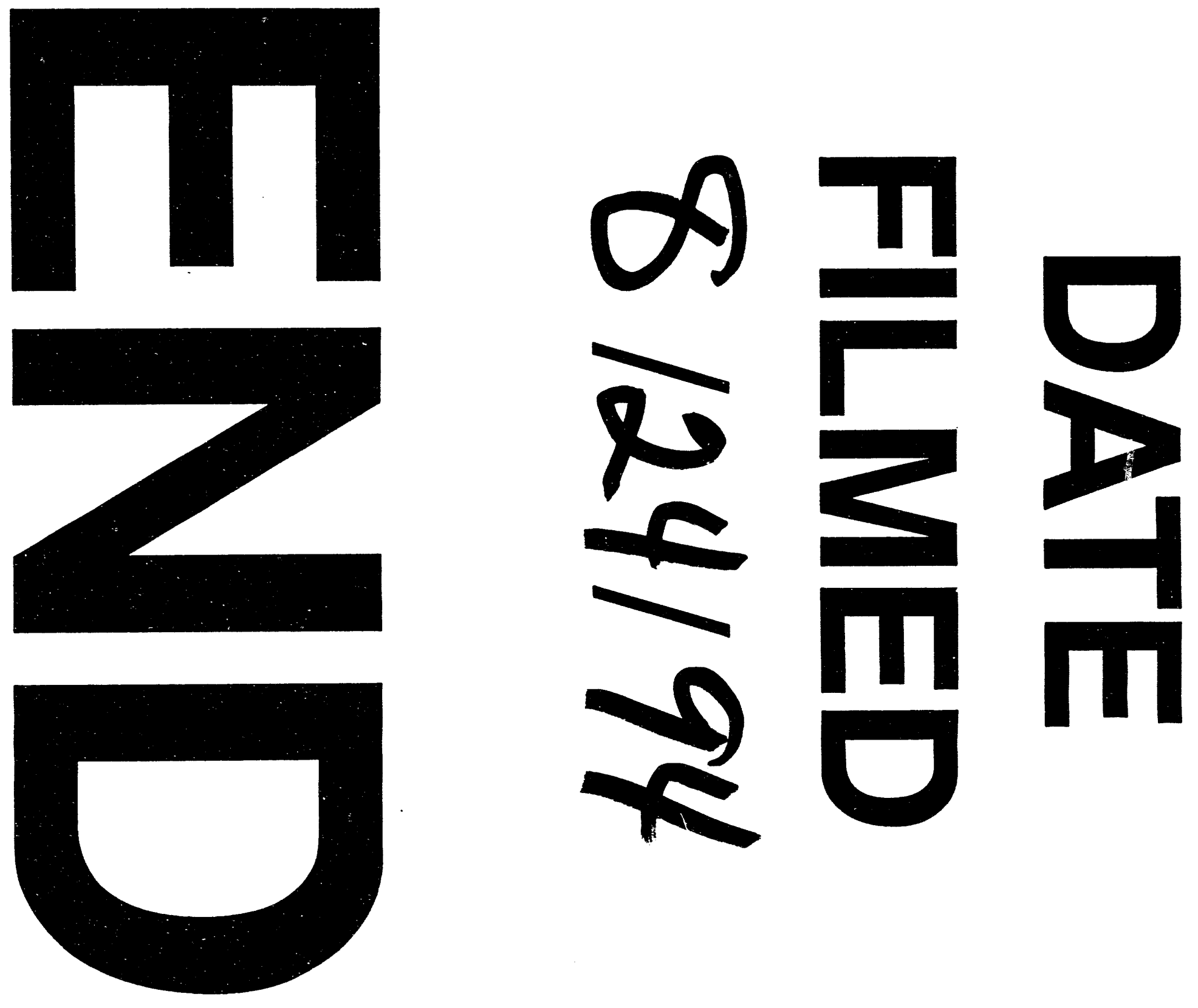
